\title{
GESTIÓN DEL CONOCIMIENTO Y POLÍTICAS DE INNOVACIÓN
}

\author{
JHONNY ANTONIO PABÓN CADAVID*
}

\section{INTRODUCCIÓN}

Toda economía es en parte una economía del conocimiento ya que no puede haber actividad económica sin conocimiento. Sin embargo, en el momento en que una sociedad, un sector o una actividad dependen intensamente de actividades basadas en el conocimiento y este se considera como un bien principal objeto del mercado es cuando esta clase de economías adquieren identidad.

En la primera mitad del siglo xx las teorías de Joseph Schumpeter destacaron que la innovación y el conocimiento son necesarios para el desarrollo económico y el crecimiento de las sociedades (Schumpeter, 1934). En la segunda mitad del siglo xx, Peter Drucker añadió que la inversión en conocimiento e innovación es un factor principal en la competitividad de los diferentes países y organizaciones, creando economías y sociedades del conocimiento (Drucker, 1969).

En las décadas recientes el conocimiento se ha considerado la fuente productiva más importante en el desarrollo económico, y juega un papel decisivo en la creación de eficiencia y prosperidad. Esta percepción es cada vez mayor en el escenario actual de la globalización, y su intensidad se refleja claramente en las empresas exclusivamente basadas en el conocimiento y su impacto en la economía (Čihovská y Hvizdová, 2011). Por ejemplo, la industria del software en Estados Unidos genera alrededor de un trillón de dólares al año, alrededor de 10 millones de empleos, el promedio del salario en la industria dobla el salario promedio de todas las otras ocupaciones y se invierten alrededor de 52 billones de dólares al año en Investigación y Desarrollo (вSA, 2016).

* Ph.D. Victoria University of Wellington, Nueva Zelanda. Magíster en Gestión del Conocimiento (MSc Knowledge Management), Nanyang Technological University, Singapur. Abogado, Universidad Externado de Colombia. Contacto: [j4207732@hotmail.com]. Fecha de recepción: 10 de octubre de 2016. Fecha de aceptación: 30 de noviembre de 2016. Para citar el artículo: Pabón Cadavid, J. A. Gestión del conocimiento y políticas de innovación. Revista La Propiedad Inmaterial n. ${ }^{\circ}$ 22, Universidad Externado de Colombia, julio-diciembre 2016, pp. 19-31. DoI: http://dx.doi.org/10.18601/16571959.n22.02. 
El consenso actual sobre las definiciones y los beneficios de la economía del conocimiento está relacionado con la intervención de las organizaciones internacionales en la elaboración del nuevo modelo de la economía global. Estas nuevas políticas generan una tendencia a desplazar la fuerza laboral, la tierra y el capital tradicional hacia la innovación y el conocimiento como factores clave de la producción (Drucker, 1998).

Una sociedad enfocada en una economía del conocimiento se caracteriza por tener una elevada proporción de industrias de alta tecnología, una importante participación del sector servicios en el mercado, un mayor porcentaje de su población ocupada en trabajos basados en el conocimiento, un uso intensivo de tecnologías de la información y la comunicación, un aumento del porcentaje de la población con altos niveles de educación, recursos humanos capacitados y comprometidos en trabajos relacionados con la investigación y desarrollo (I+D), empleos basados en la tecnología (como en la creación de software y tecnologías de la información), y un porcentaje mayor de consumidores de conocimiento (Surinder, 2009).

Las políticas de la innovación y la gestión del conocimiento cumplen un papel fundamental en el desarrollo económico de las sociedades; ellas crean y fortalecen la capacidad de colaboración entre las empresas, el sector público y las instituciones educativas, como universidades y otros centros de I+D. Adecuadas políticas y la apropiada gestión promueven nuevos modelos de competencia y cooperación, esenciales en las organizaciones para fomentar mejores resultados, que implican el uso de sistemas de conocimiento avanzados. Los ecosistemas del conocimiento y la innovación se caracterizan por la integración de múltiples modos de innovación, difusión y uso del conocimiento. Ello combina modos lineales y no lineales de innovación, lo que refleja la complejidad, la dinámica y la naturaleza adaptativa de la creación. En estos ecosistemas avanzados existe un modelo de cooperacióncompetencia que significa una compleja tolerancia a los desequilibrios y al riesgo que se produce entre diferentes redes configuradas de manera flexible y abierta (Carayannis y Campbell, 2012).

Este artículo analiza el rol de las políticas nacionales de innovación y la gestión del conocimiento para la economía, y la intersección entre uno y otro factor. Para ello estudia la reciente literatura relacionada directa e indirectamente con la gestión del conocimiento, las políticas de innovación y su relación con la economía. En primer lugar se introducen los conceptos principales que resultan relevantes para la comprensión del tema. Después, se consideran las intersecciones entre gestión del conocimiento y políticas de la innovación, y se señalan algunos modelos teóricos recientes. En la parte final se hace énfasis en el capital humano, como principal componente del capital intelectual, los activos del conocimiento y la educación. Las conclusiones consisten en una reflexión final y en algunas sugerencias para nuevas investigaciones sobre este tema. 


\section{GESTiÓN DEL CONOCIMIENTO Y POLÍticas DE INNOVACiÓN PARA EL DESARROLLO ECONÓMICO}

Conocimiento e innovación son conceptos que se deben diferenciar ya que estos coexisten y la administración de cada uno se lleva a cabo de manera diferente, aunque con puntos de convergencia. Cuando estos términos se utilizan sin distinción y en una forma intercambiable, se producen dificultades para adoptar políticas claras orientadas a su promoción (Carayannis y Campbell, 2012). Además, la forma como se defina lo que se entiende por innovación determinará el alcance de su gestión.

En las políticas relacionadas con la economía del conocimiento, la innovación suele estar relacionada con la creación o con la introducción de productos o procesos nuevos y superiores que transforman el rendimiento de los recursos en un mercado sostenible y que promueve el desarrollo económico (Carayannis y Campbell, 2012).

La innovación desde la perspectiva de las sociedades comerciales y las organizaciones empresariales es la comercialización exitosa de una invención, entrando en un mercado o creando uno nuevo, o la mejora de procesos internos y la generación de eficiencias. Para una empresa, las invenciones sólo son útiles cuando implican innovación, es decir, cuando la invención añade valor a la empresa y a la sociedad. Es pertinente señalar que en este sentido no toda invención ni necesariamente todas las patentes son parte activa en la creación de una sociedad del conocimiento (Pavitt, 1985).

La incertidumbre y el desequilibrio son características de la innovación. La incertidumbre es la fase de adopción de una innovación, en la que los mercados adoptan una invención. La incertidumbre también puede afectar a una invención que no se convierta en innovación por falta de implementación o falta de adopción en el mercado. El desequilibrio se produce cuando la invención es aceptada creando cambios, convirtiéndose en innovación, generando el desplazamiento de las tecnologías, en algunas circunstancias creando disrupciones.

La innovación es posible mediante la interacción y la gestión de las personas en las organizaciones, y es allí, en el nivel micro, donde emerge; no obstante, los contextos sociales, políticos y económicos dentro del horizonte nacional determinan y afectan el desarrollo de la innovación.

\section{I.I. Políticas de inNovación}

Las políticas nacionales de innovación pueden focalizar las competencias organizacionales necesarias para maximizar sus resultados. Esto funciona en un ciclo en donde las políticas nacionales se deben retroalimentar de la observación atenta del desarrollo de las competencias organizacionales. Así por ejemplo, la investigación en la economía de India y su relación con la gestión del conocimiento ha estado enfocada en el sector de las tecnologías de la información para la competitividad, su interacción con el desarrollo económico nacional y su relación con la economía 
internacional. En ese contexto particular, las propuestas para crear un entorno adecuado para que las empresas se integren a la economía del conocimiento se basan en la necesidad de facilitar la transferencia de información y conocimiento por medio de sistemas informáticos, bases de datos, tecnologías de la información y la interconexión de redes enteras (Chandrasekar, 2012).

Las políticas y los programas nacionales de innovación han sido establecidos para la creación y el fortalecimiento de economías del conocimiento. Países en todos los continentes han adoptado esta clase de políticas; así, en Asia, Singapur se destaca por tener una política de inversión en investigación e innovación en la que invertirá 20 billones de dólares entre 2016 y 2020 (National Research Foundation, 2016); una de las tres principales iniciativas de la estrategia de Europa 2020 es la unión en innovación (European Commission, 2010). En Oriente Medio, por ejemplo, el gobierno de Arabia Saudita en su visión 2021 plantea avanzar hacia una economía del conocimiento con la construcción de infraestructuras y competencias que impulsen la innovación como parte del interés de insertarse en la globalización de una manera sostenible a largo plazo (Emirates Competitiveness Council, 2011). La inversión de Arabia Saudita en programas de innovación y desarrollo se ha incrementado considerablemente, así como la inversión en educación de sus ciudadanos (p. ej., en el año 2015 alrededor de 200.000 saudíes estaban realizando estudios en universidades en el exterior), el fortalecimiento de sus universidades (un ejemplo notorio es la inversión en la Universidad del Rey Abdulaziz) y en herramientas informáticas dirigidas a fomentar una economía del conocimiento. Esto demuestra la implementación de políticas en Arabia Saudita con un creciente interés en el conocimiento, la innovación y la ciencia.

Es precisamente el crecimiento económico de nuevos actores en la economía global durante los últimos años, en especial de naciones fuera del contexto occidental, con gran inversión en I+D, como China e India, lo que ha llevado a una competencia económica y a demandas de conocimiento que están desafiando el modelo lineal de innovación (Carayannis y Campbell, 2012).

\section{I.2. Modelos De INNOVACIÓN}

El modelo lineal de innovación es el modelo tradicional que sigue las siguientes etapas: 1) investigación básica, 2) investigación aplicada, 3) invenciones que se mueven de las universidades a los sectores relacionados, 4) desarrollo experimental, 5) negocios y explotación en la economía. En la actualidad este modelo se enfrenta a dos grandes problemas en el contexto de la competencia global: 1) el modelo lineal se centra en el nivel nacional, y 2) este modelo no analiza cómo el conocimiento tácito de los participantes y los mercados se vuelve a conectar de nuevo a la investigación básica (Carayannis y Campbell, 2012).

Otro modelo tradicional en crisis que se usa comúnmente para comprender y promover la innovación es el modelo de triple hélice de Etzkowitz y Leydesdorff 
(2000), basado en la relación y la interacción universidad-gobierno-industria. En este modelo la interacción es no-lineal, transversal a lo nacional e intersectorial. Sin embargo, se ha demostrado que esta triple hélice es insuficiente para explicar los procesos de innovación, lo que ha llevado a numerosos autores a proponer modelos de cuatro y cinco hélices. Así, por ejemplo, el modelo de cuádruple hélice de Carayannis y Campbell resalta que la democratización de la innovación es crucial para entender la gestión en la economía del conocimiento. De acuerdo con esta propuesta, la cuarta hélice se refiere a la importancia de los usuarios, el público y la democracia para cualquier política de innovación y el conocimiento. La democratización lleva a los usuarios a crear nuevos conocimientos cuando no encuentran en los productos y servicios del mercado satisfacción a sus necesidades; un claro ejemplo es el software de código abierto creado por comunidades de usuarios que se auto-organizan y actúan glocalmente (Carayannis y Campbell, 2012)

El eje espacial de sistemas multinivel de conocimiento se compone de varios niveles de agregación: sub-nacional, nacional, transnacional, regional y global. En este eje, el concepto glocal y glocalización (globalllocal) es muy importante para comprender las intersecciones de ambos. La glocalización de la innovación es vital para medir el impacto en los contextos inmediatos donde se generan e implementan las políticas de innovación. Además, el modelo no lineal hace énfasis en cinco principios básicos: el conocimiento producido y el contexto de aplicación, la transdisciplinariedad, la heterogeneidad y diversidad organizacional, la responsabilidad social junto a la reflexividad, y el control de calidad (Carayannis y Campbell, 2012).

Todos estos paradigmas, lineal y no lineal, hélice triple y cuádruple, agrupaciones nacionales, regionales o internacionales pueden operar de manera simultánea. La forma como estos modelos se implementan en la realidad está determinada por el alcance de la gestión del conocimiento a nivel organizacional que promueve nuevos productos y procesos, incremento radical (grado de cambio estratégico) y adaptación técnico-administrativa (relación entre la innovación y los principales elementos de la organización de la empresa).

\section{GeSTIÓN DEL CONOCIMIENTO}

La gestión del conocimiento es más amplia que la gestión de la innovación, y se utiliza como un concepto global, que abarca un extenso espectro de ideas. Desde una visión amplia, la gestión del conocimiento incluye la administración que tiene como objetivo capturar, organizar, compartir, difundir y crear conocimiento formal e informal, para mejorar la productividad y para proporcionar bienes y servicios de la manera más eficiente en una organización.

Entre muchas otras herramientas y métodos para la gestión del conocimiento se destacan las comunidades de práctica, las reuniones de reflexión después de la 
acción y los cafés de conocimiento. Estas herramientas buscan la continua captura y transferencia de conocimiento producido a nivel organizacional.

La literatura dedicada a estudiar las políticas de la innovación no ha prestado mucha atención al rol de la gestión del conocimiento. Una de las razones que puede explicar esta laguna es la dificultad de conjugar las macro políticas con las decisiones a nivel micro de la gestión y administración del capital humano y de la gestión del conocimiento.

La implementación de la gestión del conocimiento (КM) debe ser parte de las políticas públicas, especialmente en los países que invierten considerablemente en ciencia y tecnología. La gestión del conocimiento se aplica tanto en organizaciones privadas como públicas con el fin de crecer dentro de un entorno que promueva la innovación y la eficiencia tanto a nivel de las organizaciones individualmente consideradas como a nivel nacional (ALHussain, Murphree y Bixler, 2012). Así por ejemplo, Singapur ha creado un ecosistema que promueve la gestión del conocimiento principalmente en el sector público con efectos en el sector privado, en especial creando competencias y capital humano alrededor de esta área (Tang, 2013). En este contexto la interpretación del término conocimiento es una parte del perfil de competencias de los recursos humanos (Surinder, 2009).

Para mejorar el poder competitivo, las empresas tienen que centrarse en la gestión del conocimiento, este es un requisito básico para organizaciones que operan dentro de un mercado basado en conocimientos, como es el caso de las universidades o de empresas de alta tecnología. Esta gestión es posible por medio de la captura, almacenamiento, análisis, interpretación, transformación, difusión y auditoría del conocimiento, ya sea como parte de las estrategias de la administración de recursos humanos o de departamentos especializados en las organizaciones.

La Recomendación n. ${ }^{\circ} 195$ sobre el Desarrollo de los Recursos Humanos de la Organización Internacional del Trabajo define las competencias como "los conocimientos, las aptitudes profesionales y el saber hacer que se dominan y aplican en un contexto específico" (Art 2b, OIT, 2004). La educación juega entonces un papel fundamental como fuente en la generación de competencias que a su vez contribuyen al desarrollo económico.

\section{I. Capital humano y COMPetencias}

La sostenibilidad del capital humano es uno de los factores más importantes que desarrollan una economía del conocimiento que sea capaz de promover la innovación y el aprendizaje continuo. Esta correlación entre el desarrollo humano, la productividad y la innovación explica los modelos exitosos de economías que han avanzado en los últimos años, como las de Singapur y Malasya, países que han invertido sustancialmente en capital humano (Ahmed y Krishnasamy, 2013). 
El capital humano marca una ventaja competitiva de primer orden (Velmurugan, 2010). Las empresas que invierten sosteniblemente en capital humano tienden a obtener una mayor rentabilidad y capacidad de adaptación. Con una adecuada gestión del conocimiento el capital humano se convertirá en activos para las organizaciones. Para avanzar en capital humano las prácticas empresariales deben identificar la existencia de determinados conocimientos en todas las fases de sus operaciones y definir las herramientas como parte de la gestión de recursos humanos (Čihovská y Hvizdová, 2011). El desarrollo del capital humano contribuye al desarrollo económico a nivel individual por medio del aprendizaje continuo, a nivel inter-organizacional por medio de la transferencia de conocimientos, a nivel social por medio de la innovación, a nivel territorial por medio del desarrollo económico regional (Trauth, 2009).

Dos obstáculos constantes para el desarrollo de capital humano son el reclutamiento adecuado de mano de obra calificada con altos niveles de educación y retener esta fuerza laboral y sus conocimientos. Estos obstáculos se presentan principalmente debido a la falta de conocimientos técnicos, competencias y habilidades en la administración de las organizaciones y en la gestión de sus recursos humanos. Además, las barreras culturales aumentan la dificultad para resolver estos obstáculos. Las barreras culturales se refieren al tipo de prácticas incompatibles o que desestimulan el aprendizaje continuo, la iniciativa, la complejidad, la creatividad, y prácticas que no reconocen la conexión entre la economía del conocimiento y la diversidad laboral, intelectual y cultural. Algunas prácticas que ayudan a solucionar estos obstáculos son: la comunicación, el trabajo en equipo, promover el liderazgo de proyectos, reconocimiento, promover el comportamiento autónomo, y la comprensión del contexto empresarial.

La evidencia empírica indica que los países con economías más robustas son aquellos cuyos trabajadores son más educados (oECD, 2016). Como se mencionó anteriormente, la innovación y los avances en la tecnología desempeñan un papel clave en el crecimiento de cualquier economía del conocimiento. El requisito fundamental para lograr este escenario es la educación, la formación de competencias y el entrenamiento. Las políticas para el desarrollo de la educación y las competencias en cualquier nación deben ser vistas desde el punto de su eficacia para impulsar a una nación hacia la economía del conocimiento. Esto implica también una democratización no solo de la educación básica sino de la educación superior que está llamada a crear nuevo conocimiento. Así, por ejemplo, Noruega posee uno de los mayores índices de democracia y al mismo tiempo un sistema de educación público de calidad y con fuerte inversión en investigación que se refleja, además, en la financiación de estudios doctorales con altos estándares internacionales (Thune et al., 2012).

Los cambios de las políticas nacionales para promover la formación por competencias, la democratización en la educación, la producción del conocimiento y una mayor inversión en el desarrollo de habilidades y de formación impactan la 
forma en que el capital humano se vincula en la generación de empleo, el crecimiento económico y el desarrollo social. La gestión del conocimiento cumple un rol fundamental articulando las políticas con las prácticas organizacionales.

\subsection{VALOR DEL CONOCIMIENTO: UN RETO PARA SU GESTIÓN}

El conocimiento que se encuentra en los trabajadores que no es compartido ni codificado corre el riesgo de perderse para la organización. Para evitar esta situación se deben crear estímulos para asegurar la transferencia del conocimiento, como la educación continua, capturar el conocimiento implícito y tácito que se encuentra en la organización, la documentación de las lecciones aprendidas de los errores organizacionales y la documentación de mejores prácticas. Estas estrategias se implementan cuando las organizaciones entienden el valor de estos conocimientos.

Las organizaciones que invierten en conocimiento lo consideran un activo vital para pensar sus dinámicas internas y su participación en mercados donde se compra y vende esta clase de activos. Los activos de conocimiento se pueden entender como un activo intangible. Dentro de la competencia global ellos juegan un papel impulsando la adaptación estratégica constante. Un gran reto de las organizaciones es medir sus activos basados en el conocimiento.

Los activos de conocimiento abarcan los derechos de propiedad intelectual. Estos activos han crecido en importancia al aportar un valor agregado significativo para las empresas y crear mercados autónomos, como las subastas de patentes (Fischer y Leidinger, 2014). Esto ha llevado a que existan nuevas herramientas de medición de los activos de conocimiento que permiten cuantificar el valor económico agregado que aportan a las organizaciones, aunque siguen existiendo retos para reportar y medir de manera eficaz, transparente y confiable el valor de estos bienes intangibles (Velmurugan, 2010). Un asunto mucho más complicado e importante en una perspectiva del largo alcance en las organizaciones es cómo evaluar y medir el capital humano. Por ejemplo, la contabilidad empresarial ha carecido de una normalización y presentación de información sobre capital humano, el cual prácticamente es inexistente en los informes contables y no se tiene en cuenta en los reportes y evaluaciones empresariales.

El reporte del capital intelectual se ha desarrollado recientemente en países con economías fuertes alrededor del conocimiento como Japón y Estados Unidos. El Instituto Americano de Contadores Públicos Certificados (AICPA) y la iniciativa global para el reporte de capital intelectual (wICI) han progresado con la creación de modelos avanzados que facilitan su medición y representación de valor en las organizaciones, lo cual a su vez promueve la gestión del conocimiento. Así por ejemplo, la definición de intangibles dentro del marco de esta iniciativa es: "recursos no físicos que generan valor a la organización en el corto, mediano y largo plazo. Los intangibles están directa o indirectamente asociados con la capacidad de la organización para crear valor. En particular ellos pueden crear dos formas 
de valor distintas pero interconectadas: estratégico y financiero" (WICI, 2016). Al momento de la caracterización económica de los intangibles, la iniciativa global reconoce que los recursos intangibles están conectados al conocimiento existente en la organización y sus personas. Es precisamente por ello que este marco reconoce que los intangibles no son necesariamente controlados o sujetos a derechos de propiedad por las organizaciones. Esta mirada supera las antiguas perspectivas reduccionistas y limitadas que solo se enfocan en la propiedad intelectual al momento de medir intangibles en las organizaciones. Esta iniciativa analiza el capital intelectual desde su triple caracterización de capital humano, capital relacional y capital organizacional.

Cuando las organizaciones entienden el capital intelectual de forma amplia, como la propuesta por WICI, adoptan estrategias para la gestión del conocimiento. Estas organizaciones se preocupan por mejorar continuamente la utilización de los conocimientos en la producción y en la prestación de servicios y por extender el uso de nuevos conocimientos a toda la organización (Čihovská y Hvizdová, 2011). Para la adecuada gestión existen diversas barreras que tienen su origen en factores humanos, tecnológicos, organizacionales y culturales. Las principales están relacionadas con la falta de apoyo de la alta gerencia a las personas o proyectos relacionados con iniciativas de gestión del conocimiento, la falta de conciencia cultural sobre la importancia de esta área, y la falta de formación y educación acerca de la gestión del conocimiento (ALHussain, Murphree y Bixler, 2012). Además, las leyes, los planes y las políticas nacionales de innovación pueden intensificar o reducir estas barreras.

Políticas que promueven la descentralización administrativa, las alianzas público-privadas, las asociaciones academia-industria, los laboratorios y centros de investigación, la educación pública y la inversión en investigación, las actividades y autonomía de las ONG y la participación comunitaria a nivel local facilitan la gestión del conocimiento.

\section{INTERSECCIÓN ENTRE MACRO-POLÍTICAS Y LA MICRO-GESTIÓN}

La gestión de la innovación tiene dos niveles de acción y ejecución, el nivel macro y el micro. El nivel macro hace referencia a las políticas y programas de orden nacional e internacional para el impulso de proyectos de innovación aunado al desarrollo de una sociedad del conocimiento. A nivel micro es la capacidad de innovación de las empresas que se refleja en sus patentes, la inversión en I+D, sus iniciativas en crear nuevos productos y procesos y la capacidad para gestionar el capital humano (Aljona y Avison, 2009).

Existen diferentes escenarios posibles para evaluar la intersección entre el nivel macro y el nivel micro de la innovación: 1 . La inexistencia de una relación; 2 . Una interacción que proviene principalmente del nivel macro que se caracteriza por las políticas públicas, pero carente de un mercado de conocimiento, con bajo nivel 
de educación y poco desarrollo de la innovación en las empresas; 3 . Una interacción que proviene desde el nivel micro que se caracteriza por la innovación de las empresas, alta participación de monopolios y oligopolios y economías de mercado con poca intervención estatal; 4. La interacción confluente de ambos niveles.

Los países ubicados en el cuarto escenario, con un alto nivel de intersección, que tienen políticas nacionales de intervención estatal en materia de innovación y un entorno de mercado apropiado, son los líderes de la sociedad del conocimiento. Esto también comporta que la implementación de la gestión del conocimiento en las organizaciones de estos países es alta. El análisis de la intersección entre los niveles micro y macro sirve para medir la eficacia de los planes estratégicos nacionales para la innovación en la promoción del desarrollo de economías del conocimiento. Además, esta clase de análisis es un buen indicador del grado de desarrollo de la gestión del conocimiento y de la manera como los planes nacionales en la práctica son adoptados por las organizaciones (Aljona y Avison, 2009).

La mayoría, si no todos, los productos incorporan múltiples tecnologías, la complejidad de los bienes y servicios, y por lo tanto el contenido de conocimiento de los productos se ha incrementado (Narula y Duysters, 2004). Esto ha llevado a niveles de especialización cada vez más profundos, pero a la vez a la interacción y colaboración constante entre competidores. En este escenario la creación de parques tecnológicos y clústeres es un buen ejemplo de la promoción de espacios de interacción entre políticas nacionales o regionales y de la gestión del conocimiento para lograr cooperación-competencia entre las organizaciones concentradas en un área.

\section{I. Clústeres y PARQues TeCNológicos}

Un clúster empresarial es un grupo de compañías cercanas geográficamente e interconectadas, cuyo propósito es proveer servicios especializados por medio de empresas de sectores afines e instituciones asociadas en campos particulares que compiten pero también cooperan (Porter, 1998). Los clústeres surgen por la necesidad de las empresas de proveer, además de sus competencias en tecnológicas básicas, nuevas competencias complementarias. Hoy en día, por lo tanto, las empresas más exitosas se unen para formar redes de innovación en todo el mundo con miras a lograr esta ventaja competitiva múltiple. Estos llamados núcleos regionales de innovación son una forma específica de crear redes y tienen un papel central en la generación de nuevas ideas innovadoras y ventajas competitivas regionales.

La unión de las competencias complementarias permite enfocar las empresas en sus propios recursos básicos; al mismo tiempo hace posible obtener los beneficios de la utilización de un excelente know-how de socios externos. Por ejemplo, en el caso de Biovalley, se trata de un clúster transfronterizo que agrupa actividades empresariales e investigación en el campo de la biotecnología. La razón del éxito del clúster Biovalley es su ubicación, que reúne tres patrones culturales diferentes. En segundo lugar, el clúster contiene varios parques biotecnológicos: Allschwil en 
Suiza, otro en Friburgo, Alemania, y uno más en Illkirch-Estrasburgo, Francia. En tercer lugar, está la creación de un Fondo Biovalley, lo que hace fácil el acceso al capital de riesgo. Por último, el clúster fue una iniciativa empresarial combinada con el apoyo del gobierno (Bureth, Pénin y Wolff, 2010).

Cabe pensar que puede haber varios factores de éxito para los clústeres, la clave está en la selección de los socios correctos en función de las habilidades adecuadas y las competencias. Una vez que los socios correctos forman una red, las empresas tienen que establecer la gestión de la red de forma sistemática, debiendo concordar entonces los vínculos de comunicación y cooperación entre ellos para la coordinación eficaz y eficiente de sus conocimientos y competencias. La gestión de la red en un clúster está basada en la cooperación con los dirigentes políticos de la región y sus principales actores económicos. Esto requiere crear una imagen de la red, el establecimiento y mantenimiento de infraestructuras, planificación, organización y financiación de los proyectos pertinentes.

Junto con la gestión de la red se debe conducir a la adaptación permanente de la visión compartida de la resolución de problemas cuando se enfrentan a riesgos e incertidumbres. El reconocimiento de estos requisitos supone facilitar y regular el proceso de comunicación entre los socios. Además, dicha metodología implica tener en cuenta que siempre se está siguiendo un conjunto de hojas de ruta que se ajustan continuamente entre ellas. Todos estos procesos se enmarcan dentro de estrategias de gestión del conocimiento.

\section{ConClusión}

La economía del conocimiento cambia la velocidad y la dirección del desarrollo económico. La intersección entre el nivel macro de las políticas de innovación y el desarrollo micro de la gestión del conocimiento es el pilar fundamental para crear capital intelectual que sea de provecho para toda la sociedad. Esta correlación es esencial para que los esfuerzos de los gobiernos y de las organizaciones estén coordinados.

Este artículo realizó un mapeo de temas que desembocan en la apertura de un espacio de reflexión alrededor de la necesidad de futuras investigaciones sobre el papel de la cultura del país y el entorno en el que se crea, comparte y comercializa el conocimiento. En este sentido, se desea invitar a pensar sobre qué tipo de capital humano, qué entorno social y qué educación son necesarios para lograr la economía del conocimiento. Además, los estudios sobre la aplicación real de las políticas de innovación en los países en desarrollo y sus barreras serán de beneficio para la comprensión de la importancia de la gestión del conocimiento desde una perspectiva glocal.

Para finalizar una recomendación básica que se debe tener en cuenta en nuestra región latinoamericana. Las políticas de innovación nacional deben promover con énfasis la movilidad del capital humano a nivel regional. Universidades y centros 
de investigación pueden incorporar la movilidad de sus profesores e investigadores como parte de estrategias dirigidas a incrementar el capital intelectual de las organizaciones. Desde este enfoque, los procesos de internacionalización de la educación y la investigación estarán inscritos en proyectos estructurados de gestión del conocimiento. Ello implica el diseño de estrategias que permitan evaluar el impacto de la movilidad en el capital relacional (p. ej., al evaluar el uso efectivo de convenios académicos), estructural (p. ej., al medir el número de publicaciones en coautoría con pares internacionales) y humano (p. ej., al analizar la adquisición de nuevas competencias) de las instituciones.

\section{BibLIOGRAFÍA}

Ahmed, Elsadig M. y Krishnasamy, Geeta (2013). "Human Capital Investment to Achieve Knowledge-Based Economy in ASEAN5: DEA Applications", Journal of the Knowledge Economy, vol. 4 (4).

AlHussain, Ahmed Z., Murphree, Edward L. y Bixler, Charles H. (2012). "Barriers to Knowledge Management in Saudi Arabia", Journal of Knowledge Globalization, vol. 5 (1).

BSA (2016). The \$1 Trillion Economic Impact of Software (Washington: BSA The Software Alliance).

Bureth, Antonine, Pénin, Julien y Wolff, Sandrine (2010). "Start-Up Creation in Biotechnology: Lessons from The Case of Four New Ventures in The Upper Rhine Biovalley", International Journal of Innovation Management, vol. 14 (2).

Carayannis, Elias G. y Campbell David F. J. (2012). Mode 3 Knowledge Production in Quadruple Helix Innovation Systems 21st-Century Democracy, Innovation, and Entrepreneurship for Development (Heidelberg: Springer).

Chandrasekar, K. (2012). "Knowledge Management to Meet the Contemporary Challenges and Charms", Advances in Management, vol. 5 (3).

Čihovská, V. y Hvizdová, Eva (2011). "Knowledge Management Formulates a New System of Wealth Creation", Economics and Management, vol. 16.

Drucker, Peter (1998). "From Capitalism to Knowledge Society", en Neef, D. (ed.), The Knowledge Economy (Woburn MA: Butterworth).

Drucker, Peter F. (1969). The Age of Discontinuity (New York: Harper \& Row).

Emirates Competitiveness Council (2011). Policy in Action: The UaE in the Global Knowledge Economy, Fast-Forwarding the Nation (Dubai: ECC).

Etzkowitz, Henry y LeydesdorfF, Loet (2000). "The Dynamics of Innovation: from National Systems and "Mode 2" to a Triple Helix of University Industry-Government Relations", Research Policy, vol. 29 (2).

European Commission (2010). EUROPE 2020 A strategy for Smart, Sustainable and Inclusive Growth (Brussels: EC).

Fischer, Timo y Leidinger, Jan (2013). “Testing Patent Value Indicators on Di- 
rectly Observed Patent Value-An Empirical Analysis of Ocean Tomo Patent Auctions", Research Policy, vol. 43 (3).

Narulaa, Rajneesh y Duysters, Geert (2004). "Globalisation and Trends in International R\&D Alliances", Journal of International Management, vol. 10 (2). National Research Foundation (2016). Research Innovation Enterprise 2020 Plan: Winning the Future through Science and Technology (Singapore: Research, Innovation and Enterprise Secretariat). OECD (2016). Education at a Glance: OECD Indicators (Paris: OECD Publishing). OIT (2004). Recomendación No. 195 sobre el Desarrollo de los Recursos Humanos: Educación, Formación y Aprendizaje Permanente (Ginebra, 92.a reunión CIT). Pavitt, K. (1985). "Patent Statistics as Indicators of Innovative Activities: Possibilities and Problems", Scientometrics, vol. 7 (1).

Porter, Michael E. (1998). "Clusters and the New Economics of Competition", Harvard Business Review, vol. 76 (6).

Schumpeter, Joseph A. (1934). The Theory of Economic Development. An Inquiry into Profits, Capital, Credit, Interest and the Business Cycle (Cambridge Mass: Harvard University Press).

Surinder, Batra (2009). "Strengthening Human Capital for Knowledge Economy Needs: an Indian Perspective", Journal of Knowledge Management, vol. 13(5). Tang, M. y RaO, M. (2013). Knowledge Management Initiatives in Singapore (Singapore: World Scientific Publishing Company).

Thune, Taran et al. (2012). PhD Education in a Knowledge Society an Evaluation of PhD Education in Norway (Oslo: Nordic Institute for Studies in Innovation, Research and Education).

Trauth, Eileen M. (2012). "Barriers to Knowledge Acquisition, Transfer and Management in Regional Knowledge Economy Development", 45th Hawaii International Conference on System Sciences (IEEE).

Velmurugan, Manivannan S. (2010). "Revisiting Accounting in the KnowledgeBased Economy", Journal of the Knowledge Economy, vol. 1 (4).

WICI (2016). WICI Intangibles Report Framework (World Intellectual Capital/ Assets Initiative).

Zorina, Aljona y Avison, David (2009). "Innovation Management in the Knowledge Economy: The Intersection of Macro and Micro Levels", en Lytras, M.D. et al. (eds.), Visioning and Engineering the Knowledge Society. A Web Science Perspective, Second World Summit on the Knowledge Society (Heidelberg: Springer). 